\title{
TEMPERATURE AND PRESSURE DEPENDENT CREEP STRESS ANALYSIS OF SPHERICAL SHELL
}

\author{
D.S. PATHANIA* \\ Department of Mathematics, Guru Nanak Dev Engineering College \\ Ludhiana, Punjab, INDIA \\ E-mail: despathania@yahoo.com \\ G. VERMA \\ Research Scholar, I.K.G. Punjab Technical University \\ Kapurthala, Punjab, INDIA
}

\begin{abstract}
In the present paper, we have studied the temperature and pressure dependent creep stress analysis of spherical shell. The review is critical to enhance the warm resistance of spherical shells in high-temperature conditions. The effect of different parameters was studied and it was noticed that the parameter $\mathrm{n}$ has a significant influence on the creep stresses and strain rates. Creep stresses and strain rates are ascertained on the premise of summed up strain measures and Seth's transition hypothesis. This investigation is completed to demonstrate the impacts of temperature on the creep stresses and strain rates in the spherical shell. The resulting quantities are computed numerically and depicted graphically. It has been watched that the spherical shell made of an incompressible material is on more secure side of configuration when contrasted with the shell made of a compressible material.
\end{abstract}

Key words: creep, temperature, pressure, spherical shell, stress, strains.

\section{Introduction}

The advancement in technology leads to development of high -temperature machinery and equipments. In order to design spherical structures under the thermo-mechanical load, the study of design aspects of structures is essential. Jayaraman et al. [1] worked on the thermal stress problem of a spherical shell under pressure by using a continuum approach. The stresses were calculated for the linear variation of temperature across the radius of the shell, and results were shown in a graphical form. Miller [2] presented a solution for stresses and displacements in a thick spherical shell subjected to internal and external pressure loads. In addition to elastic behavior, the shell material is assumed to undergo both creep and dimensional changes as the shell is pressurized. The creep behavior is initially represented with a 4-parameter linear visco-elastic model to include transient and steady-state components, then is extended to more general linear visco-elastic models. Martins [3] constructed an adequate mathematical model for explaining the conduction heat transfer phenomenon in the spherical shell surrounded by a homogeneous medium. He represented the heat transfer phenomenon by non-linear differential equations with two independent variables. Hansen et al. [4] presented the new finite volume method for solving three-dimensional thermal convection in a spherical shell under strong temperature and pressure-dependent viscosity in which the spherical shell is divided into six cubes. The model is validated by taking parameters of steady-state cubic and tetrahedral convection with other published spherical models and a detailed convergence test on successively refined grids. Song et al. [5] investigated the non-linear free vibration of a metallic rotating shallow shell under steady state temperature based on the bending theory of thin shallow shells. The partial differential equations were

\footnotetext{
* To whom correspondence should be addressed
} 
obtained to solve the two-point boundary value problem and then solved numerically by the shooting method. Kashkoli et al. [6] assumed that the thermo-creep response of the material is governed by Norton's law and an analytical solution of the problem is presented for the determination of time-dependent creep stresses and displacements of homogeneous thick-walled pressure vessels. Usmani et al. [7] presented the theoretical approach for the determination of a temperature-dependent non-linear response of shells subjected to a transverse mechanical load. The non-linear behavior of the material arises by taking into consideration the elastic-plastic behavior at high temperatures under quasi-static conditions. All the authors mentioned above have determined the solutions of the problems by considering the assumptions of creep strain laws like Norton's and the incompressibility condition, etc. These conditions are based on the classical assumptions of creep transition. These conditions are no longer valid at transition state of the solid and this state is non-linear in nature. The non-linearity in these problems can be solved by using the concept of the generalized strain measures and Seth's transition theory. Seth's transition theory can be applied to various problems of thermal creep transition. Neither the yield criterion nor the associated flow rule is assumed here. Gupta et al. [8] investigated steady state creep behavior of a functionally graded rotating disc under varying thermal gradient. The creep behavior of the FGM composite disc is described by the threshold stress-based law. Thakur et al. [9] presented a study of thermal creep stresses and strain rates in a circular disc with shaft having variable density by using Seth's transition theory. In this paper, we shall derive the results for thermal stresses under pressure in a spherical shell without resorting to any ad-hoc, semi-empirical laws and on the basis of the concept of generalized strain measures and Seth's transition theory of creep.

\section{Formulation of the mathematical problem}

Consider a thick-walled spherical shell, whose inward and outer radii are $a$ and $b$ respectively, subjected to uniform inside pressure $p$ and a temperature $\Theta$ connected at the interior surface of the shell. It is convenient to use spherical polar coordinates $(r, \theta, \phi)$, where $\theta$ the angle is made by the radius vector with a fixed axis, and $\phi$ is the angle measured round this axis. The components of displacement in the spherical co-ordinates $(r, \theta, \phi)$ are given by

$$
u=r(1-\beta), \quad v=0, \quad w=0
$$

where $u, v, w$ (displacement components); $\beta$ is the position function, depending on $r=\sqrt{x^{2}+y^{2}+z^{2}}$ only.

Generalized components of strain are given by Seth [10-11]

$$
\begin{aligned}
& e_{r r}=\frac{1}{n}\left[1-\left(r \beta^{\prime}+\beta\right)^{n}\right], \quad e_{\theta \theta}=\frac{1}{n}\left[1-\beta^{n}\right]=e_{\phi \phi}, \\
& e_{r \theta}=e_{\theta \phi}=e_{\phi r}=0
\end{aligned}
$$

where $\quad \beta^{\prime}=d \beta / d r$.

Stress-Strain Relation: The constitutive equation for stress -strain relations for a thermo elastic isotropic material is given as [12]

$$
T_{i j}=\lambda \delta_{i j} I_{1}+2 \mu e_{i j}-\xi \Theta \delta_{i j}, \quad(i, j=1,2,3)
$$

where $T_{i j}$ are the stress components, $\lambda$ and $\mu$ are Lame's constants, $I_{l}=\sum e_{k k}$ is the first strain invariant, $\delta_{i j}$ is the Kronecker delta, $\xi=\alpha(3 \lambda+2 \mu), \alpha$ is the coefficient of thermal expansion, and $\Theta$ is the 
temperature. The temperature of any part of a spherical shell under elastic-plastic stress is obtained, by virtue of the first and second law of thermodynamics. Further, $\Theta$ has to satisfy

$$
\nabla^{2} \Theta=0
$$

By using Eq.(2.2) in Eq.(2.3), the stresses are obtained as

$$
\begin{aligned}
& T_{r r}=\frac{\lambda}{n}\left[3-2 \beta^{n}-\left(r \beta^{\prime}+\beta\right)^{n}\right]+\frac{2 \mu}{n}\left[1-\left(r \beta^{\prime}+\beta\right)^{n}\right]-\xi \Theta, \\
& T_{\theta \theta}=\frac{\lambda}{n}\left[3-2 \beta^{n}-\left(r \beta^{\prime}+\beta^{n}\right)\right]+\frac{2 \mu}{n}\left[1-\beta^{n}\right]-\xi \Theta=T_{\phi \phi}, \\
& T_{r \theta}=T_{\theta \phi}=T_{\phi r}=0 .
\end{aligned}
$$

Equation of Equilibrium: The radial equilibrium of an element of the spherical shell requires

$$
r \frac{d T_{r r}}{d r}-2\left(T_{\theta \theta}-T_{r r}\right)=0
$$

where $T_{r r}$ and $T_{\theta \theta}$ are the radial and hoop stresses. For sufficiently small values of the pressure, the deformation of the shell is purely elastic.

Boundary Conditions: The temperature field satisfying the Laplace Eq.(2.4) with the boundary condition is

$$
\begin{aligned}
& \Theta=\Theta_{0} \quad \text { and } \quad T_{r r}=-p \quad \text { at } \quad r=a \text {, } \\
& \Theta=0 \quad \text { and } \quad T_{r r}=0 \text { at } \quad r=b
\end{aligned}
$$

where $\Theta_{0}$ is constant, given by Parkus [12]

$$
\Theta=\frac{\Theta_{0} a}{(b-a)}\left(\frac{b}{r}-1\right) .
$$

Critical points or turning points: Using Eqs (2.5) and (2.8) in Eq.(2.6), we get a non- linear differential equation in $\beta$ as

$$
P(P+1)^{n-1} \beta \frac{d P}{d \beta}+P(P+I)^{n}+2(1-c) P+\frac{c \xi \bar{\Theta}_{0}}{2 \mu r \beta^{n}}-\frac{2 c}{n \beta^{n}}\left[\left\{1-\beta^{n}(P+1)^{n}\right\}-\left(1-\beta^{n}\right)\right]=0
$$

where $\bar{\Theta}_{0}=-\Theta_{0} a b /(b-a), c=2 \mu / \lambda+2 \mu$ and $r \beta^{\prime}=\beta P$ ( $P$ is function of $\beta$ and $\beta$ is function of $r$ ). The transition points of $\beta$ in Eq.(2.9) are $P=0, P \rightarrow-1$ and $P \rightarrow \pm \infty$. Here, we are only interested in finding plastic stresses corresponding to $P \rightarrow-1$. 


\section{Determination of creep stresses and strain rates in spherical shell}

For finding the creep stresses and strain rates, we discuss the transition of the spherical shell at $P \rightarrow-1$. We define the transition function $R$ through principal stress difference (see Deepak [13-14], Thakur [15-16], Sharma [17], Verma [18-19] ) at the transition point $P \rightarrow-1$. The transition function $R$ is given as

$$
R=\frac{n\left(T_{\theta \theta}-T_{r r}\right)}{2 \mu}=\beta^{n}\left[(P+1)^{n}-1\right]
$$

where $R$ is a function of $r$ only .

Taking the logarithmic differentiation of Eq.(3.1) with respect to $r$ and substituting the value of $d P / d \beta$ from Eq.(2.9) and taking the asymptotic value $P \rightarrow-1$, after integration we get

$$
R=T_{r r}-T_{\theta \theta}=A_{r} r^{-2 c}\left[1-\left(1-\beta^{n}\right)\right]^{3-2 c} \exp (M)
$$

where $A_{l}$ is constant of integration, which can be determined by using the boundary condition and

$$
M=\frac{c \xi \bar{\Theta}_{0}}{2 \mu} \int \frac{d r}{r^{2}\left[1-\left(1-\beta^{n}\right)\right]} .
$$

The asymptotic value of $\beta$ as $P \rightarrow-1$ is $G / r, G$ being a constant, therefore Eq.(3.2) becomes

$$
R=T_{r r}-T_{\theta \theta}=A_{1} r^{-2 c}\left[G^{n} r^{-n}\right]^{3-2 c} \exp (M) .
$$

Substituting Eq.(3.3) into Eq.(2.6), we get

$$
T_{r r}=-2 A_{1} \int r^{-2 c-1}\left[G^{n} r^{-n}\right]^{3-2 c} \exp (M) d r+A_{2}
$$

where $A_{2}$ is a constant of integration, which can be determined by using the boundary condition. Using boundary conditions in Eq.(3.4), we get

$$
\begin{aligned}
& A_{1}=\frac{-p}{2 \int_{a}^{b} r^{-2 c-1}\left\{G^{n} r^{-n}\right\}^{3-2 c} \exp (M) d r}, \\
& A_{2}=\left[2 A_{1} \int r^{-2 c-1}\left\{G^{n} r^{-n}\right\}^{3-2 c} \exp (M) d r\right]_{r=b} .
\end{aligned}
$$

Substituting the value of constant $A_{1}$ and $A_{2}$ in Eqs (3.3), and (3.4), we get

$$
T_{r r}=-p \frac{\int^{b} r^{-2 c-1}\left\{G^{n} r^{-n}\right\}^{3-2 c} \exp (M) d r}{\int_{a}^{b} r^{-2 c-1}\left\{G^{n} r^{-n}\right\}^{3-2 c} \exp (M) d r},
$$




$$
T_{\theta \theta}=T_{\phi \phi}=T_{r r}+\frac{p r^{-2 c}\left\{G^{n} r^{-n}\right\}^{3-2 c} \exp (M)}{2 \int_{a}^{b} r^{-2 c-1}\left\{G^{n} r^{-n}\right\}^{3-2 c} \exp (M) d r} .
$$

Equations (3.5) and (3.6) give creep stresses for the spherical shell under the combined load of pressure and temperature $\Theta_{0}$. We introduce the following non-dimensional components: $R=r / b, R_{0}=a / b$, $\sigma_{r}=T_{r r} / p, \sigma_{\theta}=T_{\theta \theta} / p, G=1$, and $\alpha \Theta_{0}=\Theta_{1}$. Equations (3.5) - (3.6) in a non-dimensional form become

$$
\begin{gathered}
\sigma_{r}=-\frac{\int_{R}^{l} R^{-3 n+2 c(n-1)-1} \exp \left(M_{1}\right) d R}{\int_{R_{0}}^{l} R^{-3 n+2 c(n-1)-1} \exp \left(M_{1}\right) d R}, \\
\sigma_{\theta}=\sigma_{\phi}=\sigma_{r}+\frac{R^{-3 n+2 c(n-l)} \exp \left(M_{1}\right)}{2 \int_{R_{0}}^{l} R^{-3 n+2 c(n-1)-1} \exp \left(M_{1}\right) d r}
\end{gathered}
$$

where

$$
M_{1}=\frac{(3-2 c) \alpha \bar{\Theta}_{0}(b R)^{n-1}}{(n-1) D^{n}}
$$

Fully-Plastic State: For a spherical shell made of an incompressible material $(v \rightarrow 1 / 2$ or $C=0)$. Equations (3.7) and (3.8) become

$$
\begin{gathered}
\sigma_{r}=-\frac{\int_{R}^{1} R^{-3 n-1} \exp \left(M_{2}\right) d R}{\int_{R_{0}}^{l} R^{-3 n-1} \exp \left(M_{2}\right) d R}, \\
\sigma_{\theta}=\sigma_{\phi}=\sigma_{r}+\frac{R^{-3 n} \exp \left(M_{2}\right)}{2 \int_{R_{0}}^{l} R^{-3 n-1} \exp \left(M_{2}\right) d R}
\end{gathered}
$$

where

$$
M_{2}=\frac{3 \alpha \bar{\Theta}_{0}(b R)^{n-1}}{(n-1) D^{n}} .
$$

Estimation of creep parameters: Further the creep strain rates can be calculated for this thermo creep transition state of the spherical shell under internal pressure. For this purpose, the creep set in the strain should be replaced by creep strain rates and the stress-strain relations Eqs (2.3) become 


$$
\dot{e}_{i j}=\frac{l+v}{E} \sigma_{i j}-\frac{v}{E} \delta_{i j} T+\alpha \Theta
$$

where $\dot{e}_{i j}$ is the strain rate tensor with respect to the flow parameter $t$. Differentiating the second equation of (2.2) with respect to time $t$, we get

$$
\dot{e}_{\theta \theta}=-\beta^{n-1} \dot{\beta}
$$

For SWAINGER measure (i.e., $n=1$ ), Eq. (3.12) becomes

$$
\dot{\varepsilon}_{\theta \theta}=\dot{\beta}
$$

where $\dot{\varepsilon}_{\theta \theta}$ is the SWAINGER strain measure. From Eq. (3.1) the transition value $\beta$ is given by

$$
\beta=(n / 2 \mu)^{1 / n}\left[\sigma_{r r}-\sigma_{\theta \theta}\right]^{1 / n} .
$$

Using Eqs (3.12)-(3.14) in Eq.(3.11), we get

$$
\begin{aligned}
& \dot{\varepsilon}_{r r}=k\left[\sigma_{r r}-v\left(\sigma_{\theta \theta}+\sigma_{\phi \phi}\right)+\alpha \Theta\right], \\
& \dot{\varepsilon}_{\theta \theta}=k\left[\sigma_{\theta \theta}-v\left(\sigma_{r r}+\sigma_{\phi \phi}\right)+\alpha \Theta\right], \\
& \dot{\varepsilon}_{z z}=k\left[\sigma_{\phi \phi}-v\left(\sigma_{\theta \theta}+\sigma_{r r}\right)+\alpha \Theta\right]
\end{aligned}
$$

where $k=\left[\frac{n}{2 \mu}\left(\sigma_{r r}-\sigma_{\vartheta \vartheta}\right) E\right]^{\frac{1}{n}}$ and $\dot{\varepsilon}_{r r} \quad \dot{\varepsilon}_{\theta \vartheta}$ and $\dot{\varepsilon}_{z z}$ are strain rates tensor.

These are the constitutive equations used by Odquist [20] for finding the creep stresses and strain rates when we put $n=1 / N$.

\section{Numerical discussion about creep stresses and strain rates}

For calculating creep stresses and strain rates based on the above analysis, the following values have been taken, Poisson ratio $v=0.5$ (incompressible material $C=0$ ), and 0.2 (compressible material $C=0.75$ ), measure $n=1 / 3,1 / 5,1 / 8$ (i.e. $N=3,5,8), \alpha=5.0 \times 10^{-3} \operatorname{deg} F^{-1}, \Theta_{0}=0$ and $1000^{0} F, \Theta_{1}=\alpha \Theta_{0}=0.00$ and 5 and $D=1$. In the classical theory, measure $N$ is equal to $1 / n$. The definite integrals in Eqs (3.7)-(3.8) have been evaluated by using Simpson's rule.

Curves are plotted between creep stresses along the radii ratio $R=r / b$ (see Fig.1) for the spherical shell made of a compressible as well as of an incompressible material. It is observed from Fig. 1 that the sigma theta (hoop stress) has a maximum value at the internal surface of the spherical shell as compared to the sigma $r$ (radial stress).

For measure $n=1 / 3$ (see Fig.1a), it has been observed that the hoop stress has a maximum value at the internal surface of the shell for $C=0.75$ (compressible material) and with the introduction of the temperature this value increases further at the internal surface of the shell, whereas the value of radial stress lies between -1 to 1 due to boundary conditions for temp $=0,5$. 
For measure $n=1 / 5,1 / 8$ (see Figs $1 \mathrm{~b}, 1 \mathrm{c}$ ), it is seen that the hoop stress has a maximum value at the external surface of the spherical shell and minimum at the internal surface of shell with $C=0$ (incompressible material) and this value is maximal for $n=1 / 8$. The value of hoop stresses also increases with the temperature. The value of radial stresses lies between -1 and $l$.

In Fig.2, strain rates are produced along the radii ratio $R=r / b$ for the spherical shell made of a compressible as well as an incompressible material and notations err and eqq in Fig.2 represent strain rates $\dot{\varepsilon}_{r r}$ and $\dot{\varepsilon}_{\theta \theta}$, respectively. It is seen that strain rates are maximum at the internal surface of the spherical shell for $n=1 / 3$ as compared to $n=1 / 10$. It is observed that the strain rates decrease at the internal surface of the spherical shell with the effect of temperature for $n=1 / 3,1 / 8$. It means that the possibility of damage of the spherical shell is maximum at the internal surface for $n=1 / 3$.

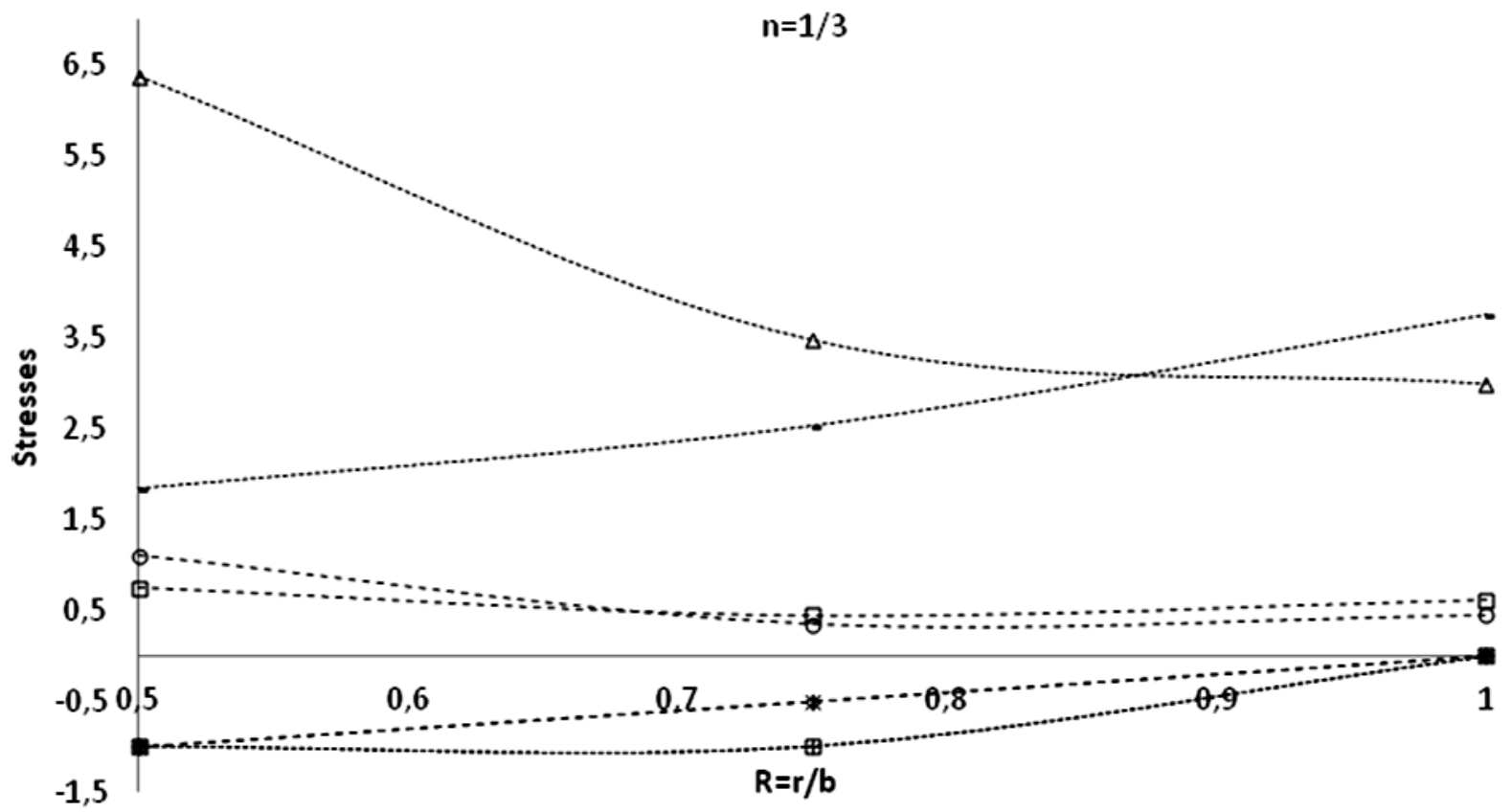

Fig.1a. Distribution of creep stresses in spherical shell under steady state temperature for $n=1 / 3$.

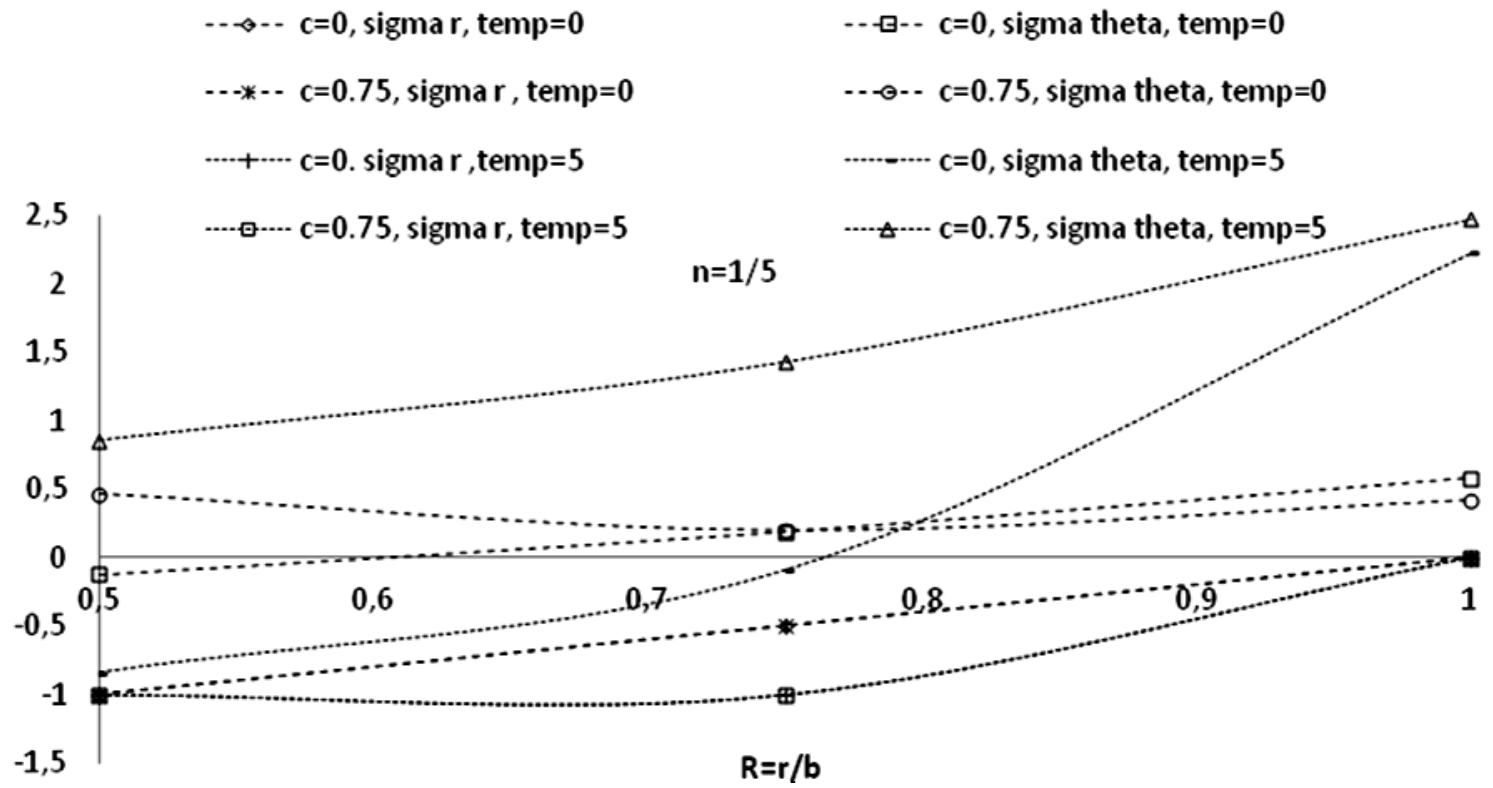

Fig.1b. Distribution of creep stresses in spherical shell under steady state temperature for $n=1 / 5$. 


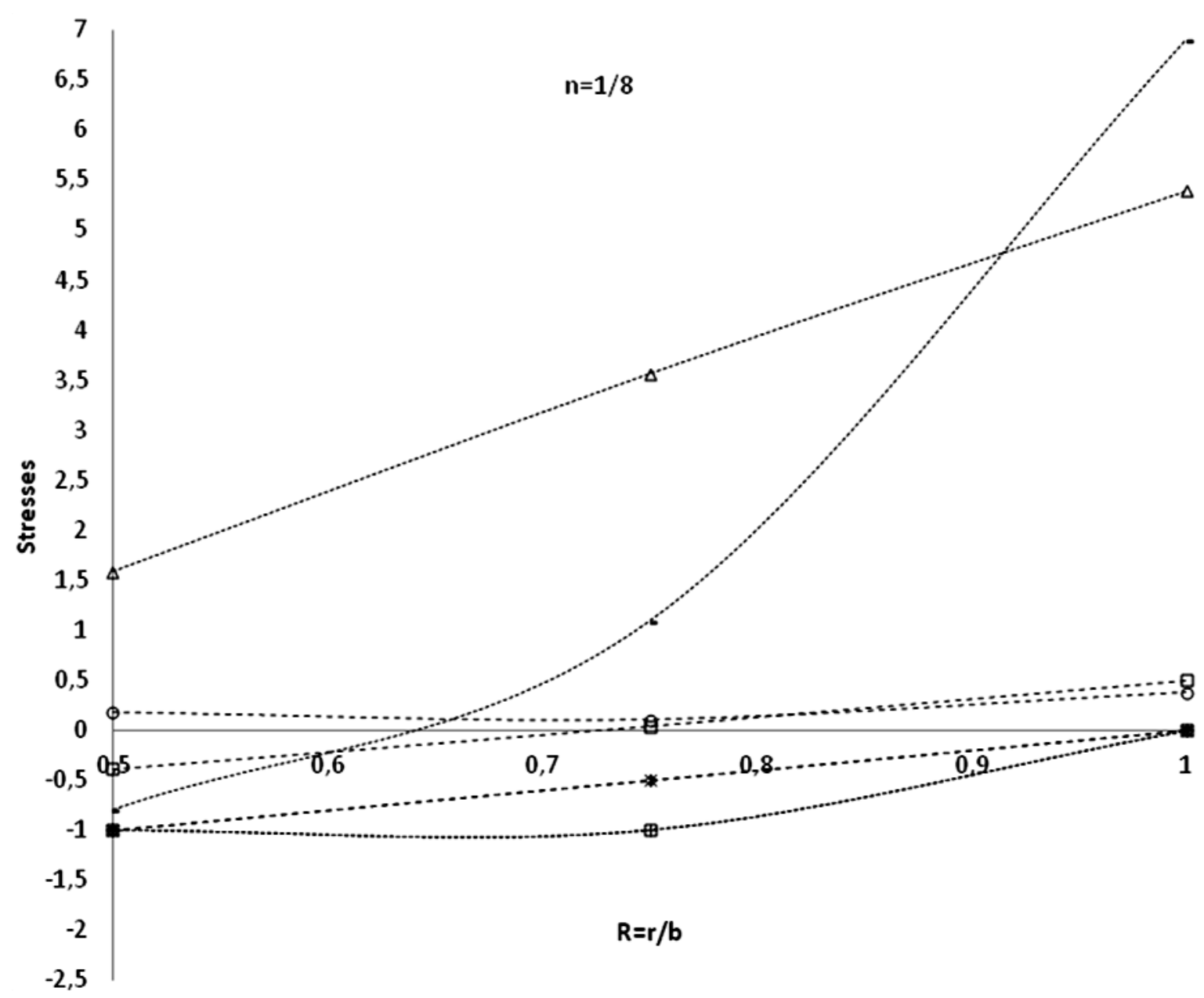

Fig.1c. Distribution of creep stresses in spherical shell under steady state temperature for $n=1 / 8$.

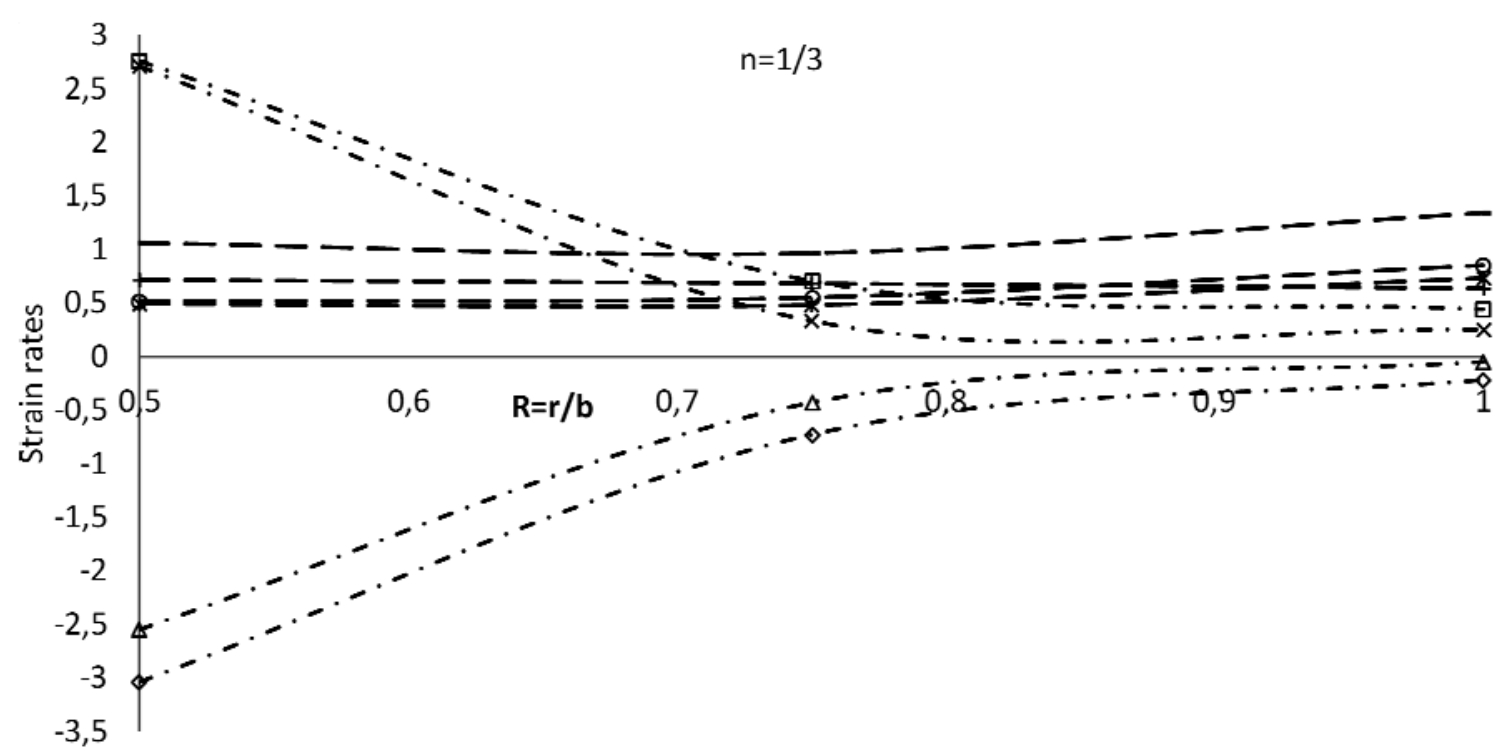

Fig.2a. Distribution of strain rates in spherical shell under steady state temperature for $n=1 / 3$. 


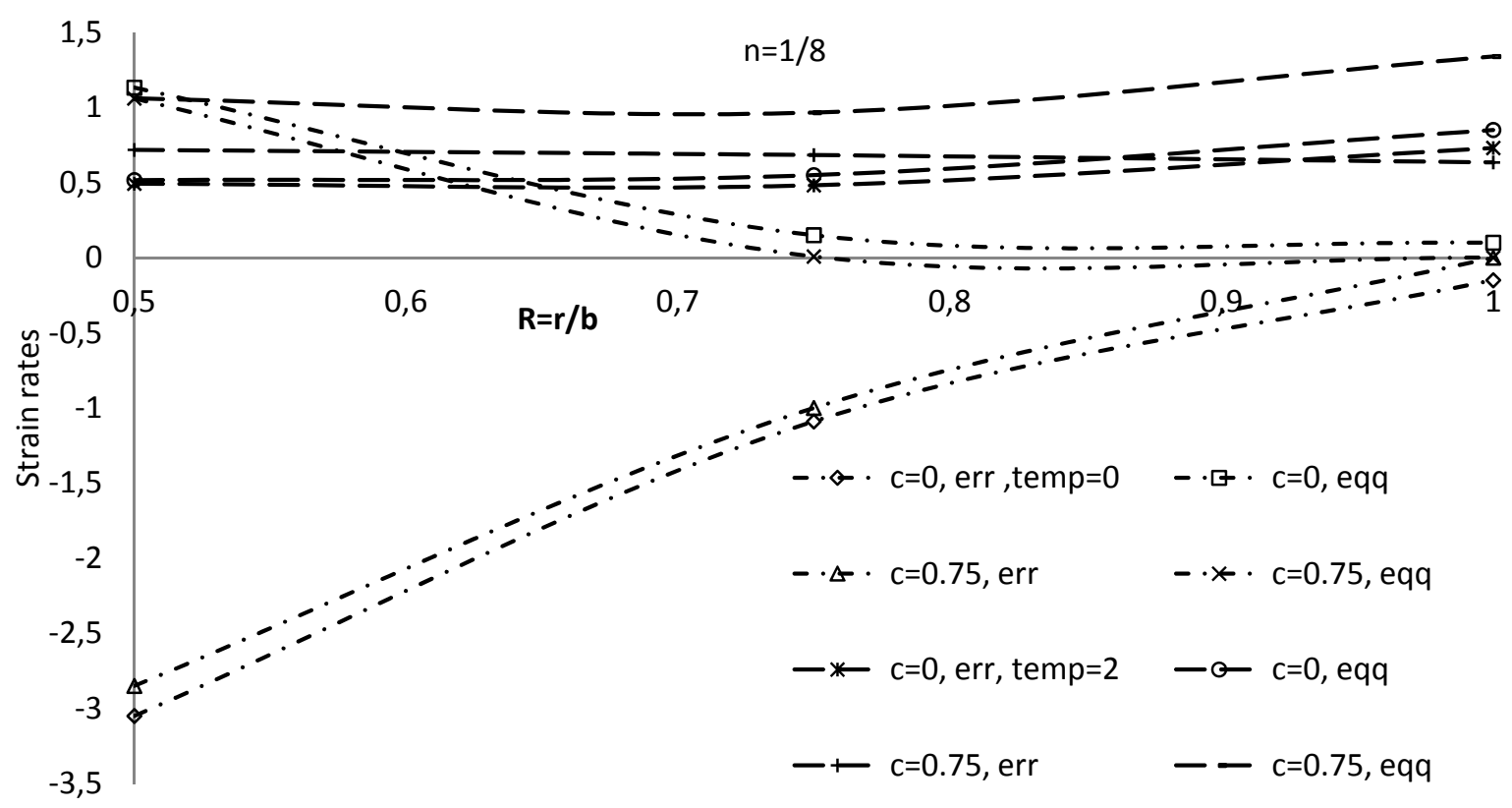

Fig.2b. Distribution of strain rates in spherical shell under steady state temperature for $n=1 / 8$.

\section{Conclusions}

In this work, an analytical solution has been obtained for creep behavior of a spherical shell under combined effect of temperature and pressure. The concept of generalized strain measures and transition theory is employed to derive the expressions for creep stresses and strain rates. The effect of different parameters is studied. It is seen that the parameter $n$ has a significant influence on the distribution of creep stresses and strain rates. The circumferential stress is found to be maximum at the internal surface and external surface for $n=1 / 3$ and $n=1 / 5,1 / 8$ respectively. This indicates that the spherical shell made up of an incompressible material for $n=1 / 8$ is on safer side of design as compared to the shell made up of a compressible material for $n=1 / 3$. The main reason is that there exist more creep strains at the internal surface of the spherical shell for measure $n=1 / 3$ and the possibility of damage increases near the interior portion of the spherical shell. It is further seen that under the effect of temperature, the significance of creep stresses is increased at the outer surface and inner surface of the spherical shell for different types of materials. It signifies that the temperature reliant materials increase the possibility of rupture at the inner surface of the spherical shell. Thus compressibility and the thermal gradient enhance the value of circumferential stresses at the internal surface of the spherical shell.

\section{Nomenclature}

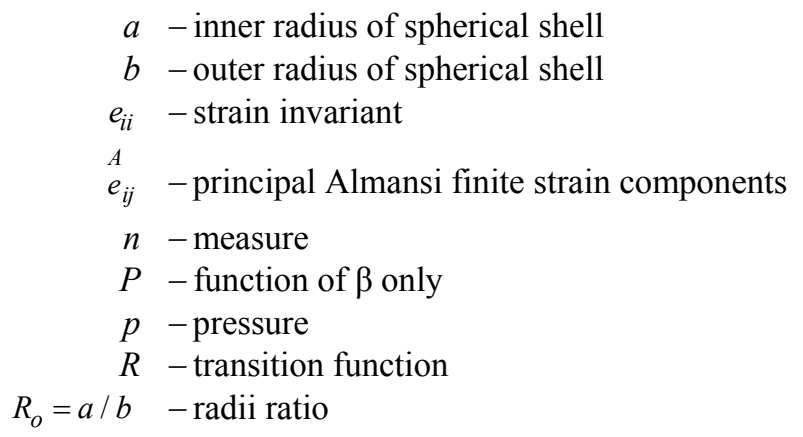




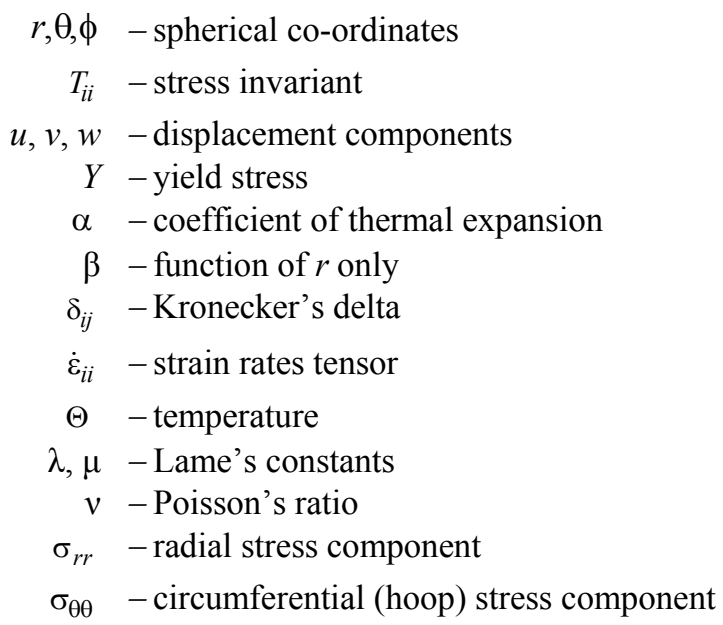

\section{References}

[1] Jayaraman J. and Rao K.P. (1978): Thermal stresses in a spherical shell with a conical nozzle. - Nuclear Engineering and Design, vol.48, No.2, pp.367-375.

[2] Miller G.K. (1995): Stresses in a spherical pressure vessel undergoing creep and dimensional changes. International Journal of Solids and Structures, vol.32, No.14, pp.2077-2093.

[3] Rogério Martins Saldanha da Gama (1997): Mathematical modeling of the non-linear heat transfer process in a gray shell surrounded by a non-participating medium. - International Journal of Non-Linear Mechanics, vol.32, No.5, pp.885-904.

[4] Stemmer K., Harder H. and Hansen U. (2006): A new method to simulate convection with strongly temperatureand pressure-dependent viscosity in a spherical shell: Applications to the Earth's mantle. - Physics of the Earth and Planetary Interiors, vol.157, No.3, pp.223-249.

[5] Wang Y. and Song H. (2010): On the nonlinear vibration of heated bimetallic shallow shells of revolution. International Journal of Mechanical Sciences, vol.52, No.3, pp.464-470.

[6] Kashkoli M.D. and Nejad M.Z. (2014): effect of heat flux on creep stresses of thick-walled cylindrical pressure vessels. - Journal of Applied Research and Technology, vol.12, No.3, pp.585-597.

[7] Khazaeinejad P. and Usmani A.S. (2016): Temperature-dependent nonlinear analysis of shallow shells: A theoretical approach. - Composite Structures, vol.141, pp.1-13.

[8] Garg M., Salaria B.S. and Gupta V.K. (2015): Modeling creep in a variable thickness rotating FGM disc under varying thermal gradient. - Engineering Computations, vol.32, No.5.

[9] Thakur P., Kaur J. and Singh S.B. (2016): Thermal creep transition stresses and strain rates in a circular disc with shaft having variable density. - Engineering Computations, vol.33, No.3.

[10] Seth B.R. (1962): Transition theory of elastic-plastic deformation, creep and relaxation. - Nature, vol.195, pp.896897.

[11] Seth B.R. (1966): Measure concept in Mechanics. - International Journal of Non-Linear Mechanics, vol.1, No.1, pp.35-40.

[12] Parkus H. (1976): Thermo-Elasticity. - New York: Springer-Verlag Wien, USA.

[13] Deepak D., Gupta V.K. and Dham A.K. (2010): Creep modeling in functionally graded rotating disc of variable thickness. - Journal of Mechanical Science and Technology, vol.24, No.1, pp.2221-2232.

[14] Deepak D., Gupta V.K. and Garg M. (2015): Creep behavior of rotating FGM disc with linear and hyperbolic thickness profiles. - Kragujevac J. Sci., vol.37, pp.35-48. 
[15] Thakur P. (2010): Creep transition stresses in a thin rotating disc with shaft by finite deformation under steady state temperature. - Thermal Science, vol.14, No.2, pp.425-436.

[16] Thakur P. (2011): Creep transition stresses of a thick isotropic spherical shell by finitesimal deformation under steady-state of temperature and internal pressure of a thick isotropic spherical shell. - Thermal Science, vol.15, No.2, pp.157-165.

[17] Sharma S., Sahai I. and Kumar R. (2013): Creep transition of a thin rotating annular disk of exponentially variable thickness with inclusion and edge load. - Procedia Engineering, pp.348-354.

[18] Gupta S. and Verma G. (2015): Creep transition of spherical shell under internal pressure. - International Scientific Journal Theoretical and Applied Science, vol.24, No.4, pp.201-207.

[19] Verma G., Rana P., Pathania D.S. and Thakur P. (2017): Creep transition in the rotating spherical shell under the effect of density variable by Seth's transition theory. - AIP Conference Proceeding 1802, 020020; doi:10.1063/1.4973270.

[20] Odquist F.K.G. (1974): Mathematical theory of creep and creep rupture. - USA: Clarendo Press, Oxford.

Received: May 26, 2017

Revised: July 18, 2018 